OPEN ACCESS

Edited by:

Maksym Yarema,

ETH Zürich, Switzerland

Reviewed by:

Adam Slabon,

RWTH Aachen Universität, Germany

Benjamin T. Diroll,

Argonne National Laboratory (DOE),

United States

${ }^{*}$ Correspondence:

Mikhail Zamkov

zamkovm@bgsu.edu

Specialty section:

This article was submitted to Nanoscience,

a section of the journal

Frontiers in Chemistry

Received: 12 June 2018

Accepted: 25 July 2018

Published: 15 August 2018

Citation:

Moroz P, Boddy A and Zamkov M (2018) Challenges and Prospects of Photocatalytic Applications Utilizing

Semiconductor Nanocrystals.

Front. Chem. 6:353.

doi: 10.3389/fchem.2018.00353

\section{Challenges and Prospects of Photocatalytic Applications Utilizing Semiconductor Nanocrystals}

\author{
Pavel Moroz ${ }^{1,2}$, Anthony Boddy ${ }^{3}$ and Mikhail Zamkov ${ }^{1,2 *}$ \\ ${ }^{1}$ The Center for Photochemical Sciences, Bowling Green State University, Bowling Green, OH, United States, ${ }^{2}$ Department \\ of Physics and Astronomy, Bowling Green State University, Bowling Green, OH, United States, ${ }^{3}$ Department of Biological \\ Sciences, Bowling Green State University, Bowling Green, $\mathrm{OH}$, United States
}

Photocatalytic systems based on colloidal semiconductor nanocrystals have gained considerable attention owing to potential benefits that include a visible-range light extinction and a low spatial overlap of photoinduced charges. When coupled to metal catalysts, nanocrystal sensitizers have demonstrated a compelling performance in homogenous photoreduction reactions, including the degradation of organic dyes and hydrogen generation. Going beyond half-cycle reactions, however, the progress in the field of nanocrystal photocatalysis has been rather limited. Here, we review some of the challenges associated with photocatalytic applications of colloidal semiconductor nanocrystals and highlight possible directions aimed toward their resolution. A particular emphasis was made on new paradigms in this field, including the possibility of harvesting triplet excitons and utilizing nanocrystal assemblies to accumulate multiple charges at the reaction site.

Keywords: photocatalysis, nanocrystals (NC), exciton dynamics, heterostructures, triplet sensitization

The prospect of employing colloidal semiconductor nanocrystals (NCs) in photocatalytic applications is inspired by unique advantages of quantum confined semiconductors over more traditional systems based on transition metal oxides (Schultz and Yoon, 2014) and precious metal coordination compounds (Concepcion et al., 2009). The benefit of inorganic nanocrystal catalysts lies in the combination of tunable redox energies and a large density of states across the visible spectrum, which gives rise to the energetic feasibility for overall water splitting (e.g., CdS, CdSe). While the photooxidation of water by semiconductor colloids has not yet been realized at a meaningful performance level, the photoreduction half-cycle reactions were shown to reach turnover numbers (TONs) in excess of $10^{5}$, which was attributed to an efficient charge separation between the semiconductor domain and an appended metal catalyst [Ni (Simon et al., 2014; Chai et al., 2016), Pt (Bao et al., 2008; Berr et al., 2010), Pd (Raza et al., 2017), or Au (Costi et al., 2008)]. Such metal-semiconductor assemblies were also deemed cost effective as most semiconductor colloids contained no precious metals with several architectures featuring heavy metal-free compositions [CuInS 2 (Zhou et al., 2017), $\mathrm{Cu}_{2} \mathrm{ZnSnS}_{4}$ (Yu et al., 2014a), $\mathrm{CuIn}_{1-\mathrm{x}} \mathrm{Ga}_{\mathrm{x}} \mathrm{S}_{2}$ (Yu et al., 2014b) NCs].

One of the early successful demonstrations of nanocrystal-based photocatalytic systems represents a heterostructured combination of a CdS nanocrystal sensitizer coupled to a $\mathrm{Pt}$ reduction co-catalyst. This architecture was shown to be up to $60 \%$ efficient in catalyzing the sacrificial reduction of protons (Costi et al., 2008; Zhou et al., 2017), and organic molecules (Brown et al., 2016; Jensen et al., 2016) under visible radiation $(\lambda \approx 450 \mathrm{~nm}$ ). 
A compelling performance of the CdS/Pt assembly was attributed to the two key aspects of this hybrid architecture: (i) the fast removal of photocorrosive holes from the CdS domain (Acharya et al., 2011), aided by electron-donating surface ligands, and (ii) a significant driving force for the electron injection into the metal catalyst (Khon et al., 2011). Further increases in the proton reduction quantum yield were made possible by employing heterostructured $\mathrm{CdSe} / \mathrm{CdS}$ and $\mathrm{ZnSe} / \mathrm{CdS}$ nanointerfaces within the sensitizer component (Zhu et al., 2012; Kalisman et al., 2016), which allowed increasing the spatial extent of the photoinduced charge separation between hole-rich (CdSe, $\mathrm{ZnSe}$ ) and electron-rich (Pt) domains (see Figures 1a-c; HewaKasakarage et al., 2009; O’Connor et al., 2012; Kalisman et al., 2016).
While Pt remains to be the most efficient co-catalyst, other compounds have also been explored in combination with semiconductor colloids to drive sacrificial reduction reactions. For instance, Ni-based heterostructures comprising a CdS sensitizer have enabled up to $50 \%$ of the $\mathrm{H}_{2}$ production internal efficiency (Simon et al., 2014; Zhukovskyi et al., 2015). Non-noble metal co-catalysts, including $\mathrm{Ni}_{2} \mathrm{P}, \mathrm{Co}_{2} \mathrm{P}, \mathrm{FePt}$ metal phosphides, and bimetallic compounds appended to CdS nanocrystals, have also been investigated as an alternative to Pt (Cao et al., 2014, 2015; Cheng et al., 2016). Regarding the sensitizer component, attempts to reduce the $\mathrm{Cd}$ content have led to its partial replacement by $\mathrm{Cu}$ in $\mathrm{Cu}_{1.94} \mathrm{~S}-\mathrm{Zn}_{\mathrm{x}} \mathrm{Cd}_{1-\mathrm{x}} \mathrm{S}$ heteronanorods (Chen et al., 2016), or a complete removal of Cd in CZTS/Pt heterostructures (see Figure 1e). Photocatalytic applications of

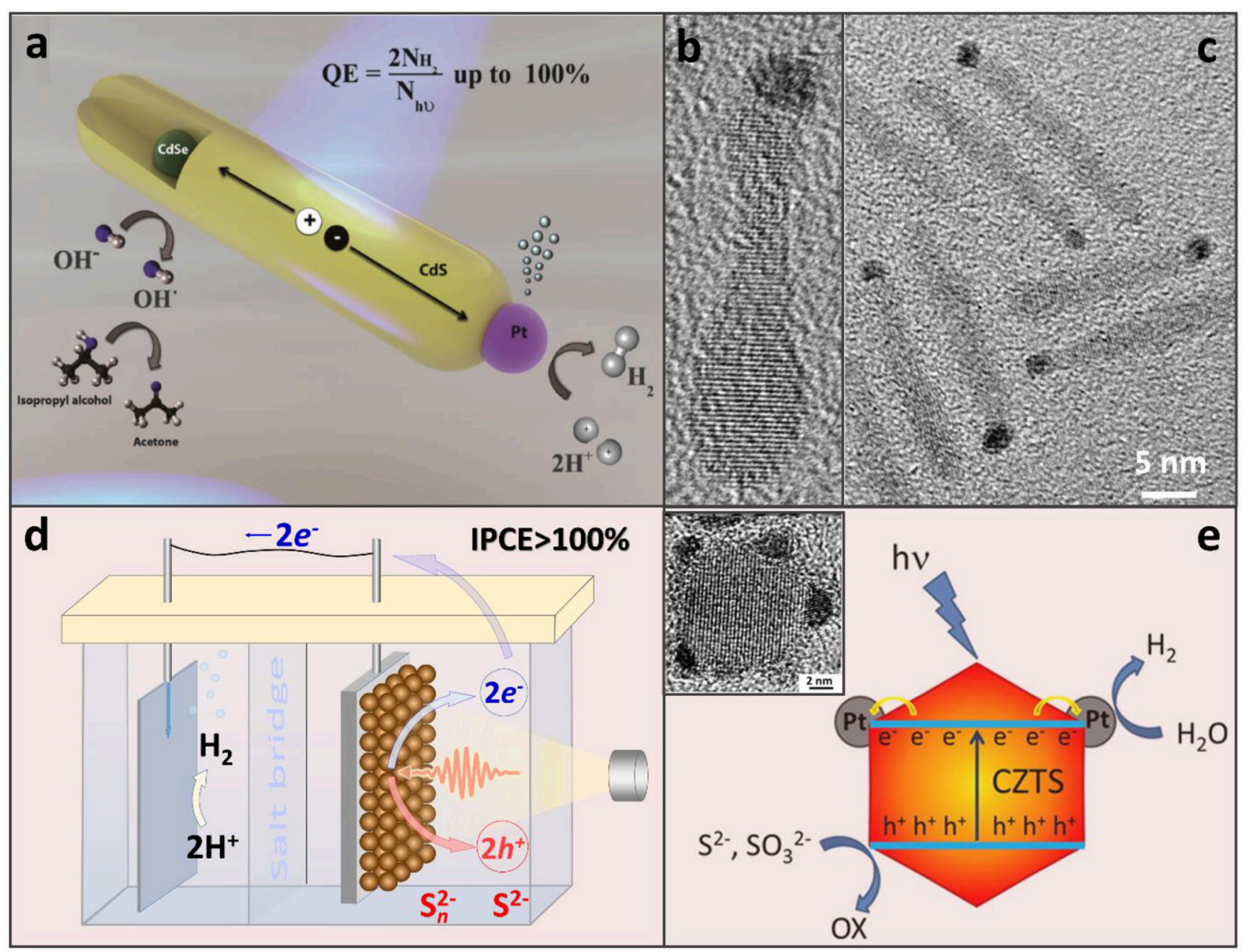

FIGURE 1 | (a) CdSe/CdS nanorods appended with a Pt co-catalyst that serves the role of an electron sink for the accumulation of photoinduced electrons at a single reductive site. Adapted with permission from Kalisman et al. (2016). Copyright 2016 American Chemical Society. (b,c) TEM images of CdSe/CdS nanorods selectively tipped with a Pt catalyst on one side. Based on the location of the CdSe bulb, Pt appears to grow on an opposite end. Adapted with permission from Khon et al. (2013). Copyright 2016 American Chemical Society. (d) Photoelectrochemical hydrogen evolution from aqueous $\mathrm{Na}_{2} \mathrm{~S}$ solution with over $100 \%$ of incident photon-to-current efficiency (IPCE). PbS NC-based active region of the working electrode (right, brown spheres) where one photon may be converted into two electron-hole pairs via MEG (Yang et al., 2014). Energized electrons are then transferred to the dark electrode (left) for $\mathrm{H}_{2}$ production and sulfide ions are oxidized by the holes. (d) Illustration of the $\mathrm{H}_{2}$ evolution on CZTS-Pt heterostructured nanoparticles in the presence of $\mathrm{S}^{2-}$ and $\mathrm{SO}_{3}^{2-}$ hole scavengers. Adapted with permission from Yu et al. (2014a). 
semiconductor nanocrystal assemblies have been attempted as well (Warren et al., 2013; Yang et al., 2014). As an interesting example of an emerging paradigm, photoelectrodes comprising $\mathrm{PbS}$ semiconductor nanocrystal solids were shown to generate multiple photoinduced electrons-hole pairs from a single UV exciton, a phenomenon known as the multiple exciton generation (MEG) (Yan et al., 2017; Zamkov, 2017). A particular advantage of the MEG effect in photoelectrochemical cells over the same phenomenon in photovoltaic devices (Beard et al., 2013) was the reverse anode architecture that did not filter the excitation light through the oxide hole-blocking layer (Yan et al., 2017).

Many years of extensive research on photocatalytic applications of semiconductor nanocrystals has identified the key performance-limiting factors of these systems, most of which could be traced to the instability of inorganic semiconductors under catalytic conditions. Depending on a particular material, the catalytic performance of corresponding nanocrystals was shown to suffer from such issues as photocorrosion, a short excited state lifetime, poor colloidal stability in acidic solutions, or a limited ability to convey the photoinduced charges to active catalytic sites.

Photocorrosion of the semiconductor material due to the accumulation of photoinduced holes represents one of the most significant issues impeding photocatalytic applications of colloidal nanocrystals. Since chalcogenides are readily oxidized (Kamat et al., 2014), nanocrystal catalysts comprising CdS, CdSe, CZTS, or PbS semiconductors eventually undergo some degree of the chemical degradation, particularly when positive charges are not regenerated quickly. Even if the timely regeneration of holes is achieved via sacrificial agents, the photooxidizing energy could still be transferred to surface ligands triggering their desorption and the subsequent nanoparticle aggregation (Acharya et al., 2011; Hines and Kamat, 2014). For instance, commonly used mercaptopropionic (MPA) or mercaptoundecanoic (MUA) acids ligands are readily oxidized by scavenged holes resulting in the formation of disulfides. Consequently, these thiolates need to be continuously replaced in order to sustain the reduction half-cycle rate. One effective strategy is to use an abundant concentration of the scavenger moiety in solution (e.g., ascorbic acid) (Han et al., 2012) that quickly relieves the nanocrystal-ligand system of a positive charge.

Semiconductor photocorrosion could also be the result of defective surfaces that tend to localize positive charges at potential energy minima (Utterback et al., 2016). It was shown that holes diffuse through such defects by hopping, thus creating hot spots for chalcogenide oxidation and other side reactions. The localization of holes inside the nanocrystal sensitizer was also shown to reduce the ensuing catalytic activity due to a low sacrificial regeneration rate (Utterback et al., 2016). For instance, such a confinement of holes may be responsible for a relatively low photocatalytic activity of $\mathrm{CuInS}_{2} \mathrm{NCs}$ where these charges become trapped on $\mathrm{Cu}^{+}$ions within the lattice structure (Leach and Macdonald, 2016; Fuhr et al., 2017).

In addition to photocorrosion, the performance of nanocrystal-based photocatalytic systems could, in some cases, be limited by short lifetimes of singlet excitons. Indeed, homogenous systems utilizing organometallic dyes benefit from an effective way of storing the photon energy through a rapid intersystem crossing into a triplet state (McCusker and Castellano, 2016; Twilton et al., 2017), which lifetime can extend into hundreds of microseconds [e.g., the triplet state of $\operatorname{Ir}(p p y)_{3}$ ] (Hofbeck and Yersin, 2010). The radiative lifetimes of singlet excitons in cadmium chalcogenide nanocrystals, on the other hand, are in the 10-100 ns range, which requires the dissociation of excitons on a faster time scale. In practice, the temporal window for a photoinduced charge transfer to a catalyst is even shorter due to competing pathways of non-radiative exciton dissociation at trap states, caused by the increased density of dangling bonds in aqueous environments.

The task of increasing excited state lifetimes of colloidal nanocrystals could be accomplished both by extending radiative lifetimes and reducing the density of charge-localizing trap states. In regard to the former condition, some groups have employed a heterojunction of the two semiconductor materials exhibiting a type II band edge alignment at the interface, which increases the spatial separation of photoinduced charges (Amirav and Alivisatos, 2010). The benefits of this strategy were demonstrated through the observation of a nearly $100 \%$ quantum yield (QY) for $\mathrm{MV}^{2+}$ photo reduction in Pt-tipped CdSe/CdS nanorods (Zhu et al., 2012) vs. a 60\% QY observed for in Pt-tipped CdS structures (Bao et al., 2008). In addition to CdSe/CdS semiconductor combination, type II heterojunctions utilizing $\mathrm{ZnSe} / \mathrm{CdS}$ (O'Connor et al., 2012) and $\mathrm{Cu}_{1.94} \mathrm{~S}-\mathrm{Zn}_{\mathrm{x}} / \mathrm{Cd}_{1-\mathrm{x}} \mathrm{S}$ (Chen et al., 2016) semiconductors have also been shown to enhance excited state lifetimes beyond those of single-phase nanocrystals. One potential issue with employing such type II interfaces in photocatalytic applications concerns the fact that one of the separated charges resides in the enclosed domain, which is shielded from the external environment by the other material (e.g., a core/shell or dot-in-a-rod geometry) (Perera et al., 2012). As a result, the confined carrier cannot be efficiently regenerated. In order to expose both semiconductor domains of a type II heterostructure to a redox environment, chemical etching could be employed. It was shown that etching of spatiallyasymmetric $\mathrm{CdSe} / \mathrm{CdS}$ nanorods results in the formation dimerlike structures where both donor and acceptor components are in direct contact with the external environment, resulting in the increased catalytic activity (Khon et al., 2013).

One emerging strategy for enhancing radiative lifetimes of excitons in nanocrystal-based photocatalytic systems relies on doping of semiconductors with transition metal ions. Lattice incorporated $\mathrm{Mn}^{2+}$ or $\mathrm{Cu}^{+}$can serve as hole trap sites promoting exciton localization in the bulk of the nanocrystal. In colloidal solutions, this strategy can allow extending excited state lifetimes into a microsecond range for $\mathrm{Ag}^{+}-$and $\mathrm{Cu}^{+}$doped CdSe NCs (Kholmicheva et al., 2017; Nelson et al., 2017), or even a millisecond range for $\mathrm{Mn}^{2+}$-doped $\mathrm{ZnSe} / \mathrm{ZnS}$ core/shell ( $\mathrm{Pu}$ et al., 2016) and CdS QDs (Knowles et al., 2015). The photocatalytic applications of doped semiconductor nanocrystals, however, have not yet been explored. A potentially adverse aspect of this architecture concerns the slow regeneration of photoinduced holes localized at dopant sites. Another possible strategy for increasing the nanocrystal excited state lifetime is based on reducing the density of surface traps. 
The two approaches that were shown successful in this regard have employed either exciton-delocalizing ligands or a defect-passivating semiconductor shell (Grenland et al., 2017). The former strategy was recently demonstrated through the employment of hole-accepting ligand molecules that were covalently linked to nanocrystals via a thiolate binding group (Ding et al., 2015; Olshansky et al., 2015). By using ferrocene ligands with different alkyl chain lengths it was possible to find an optimal driving force for hole removal. As a result, existing surface traps could be mitigated without compromising the ability to extract photoinduced charges. Similarly, surface defects can be neutralized through the use of exciton delocalizing PZT ligands that were shown to scavenge holes on a picosecond to nanosecond time scale in CdS and CdSe NCs (Wu et al., 2015; Lian et al., 2016).

An important prerequisite of any photocatalytic system is the ability to accumulate photoinduced charges at an active site. This is particularly relevant in the case of multi-electron reactions where charges need to be collected onto a single catalytic domain. In regard to semiconductor sensitizers, this aspect was experimentally confirmed through the observation of a diminishing hydrogen production efficiency in Pt-decorated CdSe@CdS rods with the increasing number of appended Pt catalysts (Nakibli et al., 2015). In particular, nanorods tipped with a single Pt domain showed $\sim 1.6$ times the efficiency for the $\mathrm{H}_{2}$ production as compared to nanorods containing two Pt domains. This result was attributed to the competition of the two metal tips for photoinduced electrons absorbed by a shared semiconductor domain. The importance of funneling the absorbed energy to a catalytic site was also illustrated by an earlier study (Amirav and Alivisatos, 2010) showing an increased $\mathrm{H}_{2}$ production rate by Pt-tipped CdS nanorods with an increasing CdS length.

The above mentioned benefits and drawbacks of nanocrystalbased photocatalytic systems, identified and investigated by the community over the course of many years, allow formulating design principles for the future development in this field. The key challenges to be addressed include the suppression of photocorrosion by photoinduced holes, the reduction of the trap states' effect, maintaining a suitable $\mathrm{pH}$ balance to prevent aggregation in acidic buffers, and funneling the excitation energy to an active reaction site (Aldana et al., 2005). Below we would like to discuss the design of the two emerging nanocrystal photocatalytic platforms that show strong potential for overcoming the aforementioned challenges. The first is based on a recently demonstrated ability of semiconductor nanocrystals to harvest triplet excitons through the Dexter energy transfer to coordination compounds exhibiting long-lived excited states. The second approach utilizes closed-coupled nanocrystal assemblies to funnel the absorbed energy to a catalytic site. Below we described the two paradigms in more detail.

The demonstrated ability of semiconductor nanocrystals to harvest triplet excitons (Mongin et al., 2016) offers new opportunities in light sensitization of photoinduced redox reactions (Figure 2a). By engaging in the Dexter energy transfer with molecular photoredox catalysts, such as $\left[\mathrm{Ru}(\mathrm{bpy})_{3}\right]^{2+}$ or $\operatorname{Ir}\left(\right.$ ppy) ${ }_{3}$ coordination compounds (Arias-Rotondo and McCusker, 2016), nanocrystal energy could be transformed into
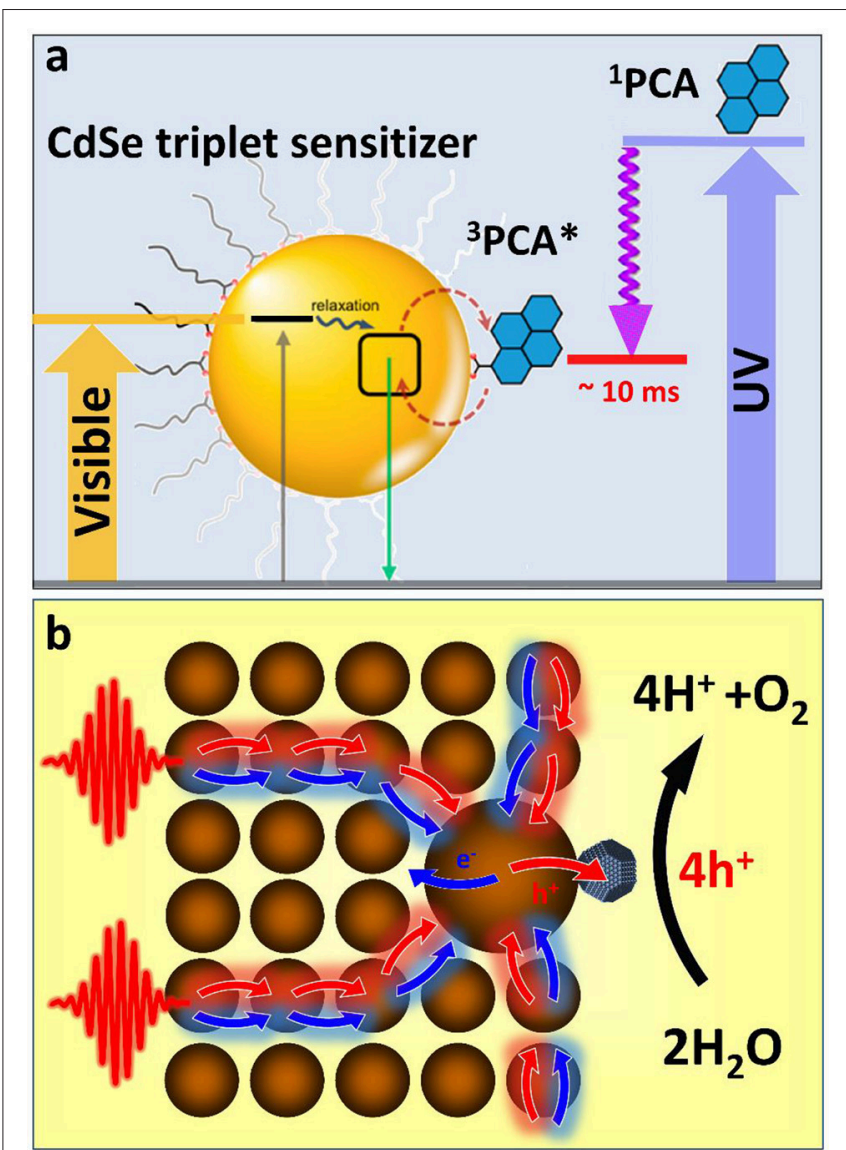

FIGURE 2 | (a) Triplet sensitization of 1-pyrenecarboxylic acid (PCA) molecular acceptor by CdSe NCs via photoinduced triplet-triplet energy transfer (TTET). Close to $90 \%$ of photons absorbed by the semiconductor QD could be stored in form of long-lived triplet states of ${ }^{3} \mathrm{PCA}^{*}$. The associated exciton energy loss resulting from TTET is less than $20 \%$, which compares favorably with a $>50 \%$ energy loss accompanying ${ }^{1} \mathrm{PCA} \rightarrow{ }^{3} \mathrm{PCA}$ intersystem crossing. Adapted with permission from Garakyaraghi and Castellano (2018). Copyright 2016 American Chemical Society. (b) A proposed scheme for funneling the photoinduced energy in assemblies of semiconductor nanocrystals via the diffusion toward the low-energy gap reaction center. This strategy benefits multi-electron catalytic processes by increasing the probability of multiple charges to be collected on the same catalytic site.

a long lived state with minimal energy losses. While such energy transfer has been demonstrated for simple molecules, such as ruberene (Wu et al., 2016) or 9-anthracenecarboxylic acid (ACA) (Mongin et al., 2016), sensitizations of triplet excited states of photoredox coordination compounds is imminent. Of a particular interest are metal polypyridyl complexes, which exhibit excellent oxidizing and reducing properties. For instance photoexcited $\left[\mathrm{Ru}(\mathrm{bpy})_{3}\right]^{3+}$ can oxidize water into $\mathrm{O}_{2}$ and protons via a metal oxide catalyst (Hara et al., 2000), while, $\left[\mathrm{Ru}(\mathrm{bpy})_{3}\right]^{2+*}$ triplet states can be utilized for reducing methylviologen (via ligands), a recyclable carrier of electrons.

Employing semiconductor nanocrystals as triplet sensitizers of photoredox coordination compounds would allow avoiding many aforementioned issues of nanocrystal photocatalytic materials related to photocorrosion, hole regeneration, and 
short singlet lifetime. On the other hand, coupling nanocrystal sensitizers to organometallic catalysts will extend the usable portion of the solar spectrum into the visible range. This is because the excitation of a triplet state undergoes via a photon absorption into a singlet metal-ligand charge transfer state $\left({ }^{1}\right.$ MLCT) followed by a rapid intersystem crossing to a ${ }^{3}$ MLCT state, which is commonly accompanied by an $\sim 1 \mathrm{eV}$ energy loss (due to large splitting of singlet and triplet states). Since such singlet-triplet splitting in semiconductor nanocrystals is usually much smaller (within thermal $\mathrm{kT} \sim 30 \mathrm{meV}$ ), the associated energy loss will be reduced. Furthermore, a molar absorptivity of CdSe at $400-450 \mathrm{~nm}$ is 20 times greater (Yu et al., 2003) than that of the ${ }^{1}$ MLCT transition in $\left[\mathrm{Ru}(\mathrm{bpy})_{3}\right]^{2+}\left(\sim 13,000 \mathrm{M}^{-1}\right.$ $\mathrm{cm}^{-1}$ in acetonitrile). Considering that the efficiency of triplet exciton transfer from CdSe to organic acceptors, such as ACA is over 90\% (Mongin et al., 2016), there is an expected benefit in employing semiconductor nanocrystals for sensitizing redox reactions.

Like any excitonic system, an assembly of semiconductor nanocrystals can be designed to transfer the photoinduced energy from the excitation site toward the potential energy minima through the process of exciton diffusion. Such funneling of the photoinduced energy is often utilized by biological systems as a mechanism to drive multiple carriers to the reaction center where multielectron catalytic reactions can subsequently take place. For instance during the oxygenic photosynthesis in plants, light is absorbed by hundreds of pigments (e.g., chlorophylls) that transfer the photoinduced energy to a small number of special pigments (P680), which are capable of charge separation (Blankenship, 2013). P680 will then share a photoinduced hole with a water-oxidizing complex (WOC) (Najafpour et al., 2017). After four oxidizing equivalents have been stored at the WOC site, it obtains four electrons from water molecules causing $\mathrm{H}_{2} \mathrm{O}$ splitting. We expect that nanocrystal assemblies could be employed in a similar manner in order to drive multielectron catalytic processes, such as water oxidation or hydrogen production. For instance, the diffusion of excitons in a nanocrystal solid to a nanoparticle with the smallest band gap (Kholmicheva et al., 2015) (an equivalent of the P680 pigment in PSII) can be used to collect multiple excitons in the same spot. The accepting dot could be appended with a catalyst that would assist the charge separation to store photoinduced charges. The presence of an electron- (or hole-) accepting

\section{REFERENCES}

Acharya, K. P., Khnayzer, R. S., O’Connor, T., Diederich, G., Kirsanova, M., Klinkova, A., et al. (2011). The role of hole localization in sacrificial hydrogen production by semiconductor-metal heterostructured nanocrystals. Nano Lett. 11, 2919-2926. doi: 10.1021/nl20 $1388 \mathrm{c}$

Aldana, J., Lavelle, N., Wang, Y., and Peng, X. (2005). Size-dependent dissociation $\mathrm{pH}$ of thiolate ligands from cadmium chalcogenide nanocrystals. J. Am. Chem. Soc. 127, 2496-2504. doi: 10.1021/ja047000+

Amirav, L., and Alivisatos, A. P. (2010). Photocatalytic hydrogen production with tunable nanorod heterostructures. J. Phys. Chem. Lett. 1, 1051-1054. doi: $10.1021 /$ jz100075c catalysis would also allow avoiding the multiexciton populations on a single nanocrystal, which are subject to a rapid decay through Auger recombination. Such nanocrystal assembly could be incorporated into a photoelectrochemical cell (see Figure 1d) or even harnessed within an "artificial leaf" platform (Liu et al., 2016). The key advantage of this architecture lies in the sequential collection of multiple charges within the same catalytic complex (Figure 2b). Zero-dimensional nanocrystals in these assemblies could be substituted with either one- or two-dimensional nanostructures in order to increase the energy transfer efficiency and reduce Auger recombination rates. Notably, without such an energy "antenna," nanocrystal-based water splitting systems would exhibit very low efficiencies (Kalisman et al., 2015) even when designed with a robust corrosion suppression mechanism.

In summary, the prospect of employing semiconductor nanocrystals in photocatalysis offers a number of unique benefits related to a spatially-extended charge separation and visible-range light absorption, which have been confirmed through a compelling performance in reduction half-reactions. In order for such systems to become practical, however, additional challenges need to be resolved. These pertain to the semiconductor photocorrosion, short excited state lifetimes, and poor control over energy transfer to catalytic sites. To resolve these issues, several emerging strategies have been proposed and discussed. Among potential solutions, harnessing nanocrystals as triplet sensitizers of photoredox coordination compounds is expected to enhance the absorption characteristics of the latter while decreasing the damage of the semiconductor. Assemblies of inorganic colloids can also be used for funneling the photoinduced energy to reactive sites in a manner analogs to the action of chlorophylls in PSII. This geometry could inspire a cascade like design of photosynthetic assemblies for water oxidation.

\section{AUTHOR CONTRIBUTIONS}

All authors have contributed equally to preparing the review article.

\section{ACKNOWLEDGMENTS}

We gratefully acknowledge OBOR Material Networks program and NSF Award CHE-1465052 for financial support.

Arias-Rotondo, D. M., and McCusker, J. K. (2016). The photophysics of photoredox catalysis: a roadmap for catalyst design. Chem. Soc. Rev. 45, 5803-5820. doi: 10.1039/C6CS00526H

Bao, N., Shen, L., Takata, T., and Domen, K. (2008). Self-templated synthesis of nanoporous CdS nanostructures for highly efficient photocatalytic hydrogen production under visible light. Chem. Mater. 20, 110-117. doi: $10.1021 / \mathrm{cm} 7029344$

Beard, M. C., Luther, J. M., Semonin, O. E., and Nozik, A. J. (2013). Third generation photovoltaics based on multiple exciton generation in quantum confined semiconductors. Acc. Chem. Res. 46, 1252-1260. doi: 10.1021/ar3001958

Berr, M., Vaneski, A., Susha, A. S., Rodríguez-Fernández, J., Döblinger, M., Jäckel, F., et al. (2010). Colloidal CdS nanorods decorated with subnanometer sized 
Pt clusters for photocatalytic hydrogen generation. Appl. Phys. Lett. 97:093108. doi: $10.1063 / 1.3480613$

Blankenship, R. E. (2013). Molecular Mechanisms of Photosynthesis. Malden, MA; Oxford: Blackwell Science.

Brown, K. A., Harris, D. F., Wilker, M. B., Rasmussen, A., Khadka, N., Hamby, H., et al. (2016). Light-driven dinitrogen reduction catalyzed by a CdS:nitrogenase $\mathrm{MoFe}$ protein biohybrid. Science 352, 448-450. doi: 10.1126/science. aaf2091

Cao, S., Chen, Y., Hou, C. C., Lv, X. J., and Fu, W. F. (2015). Cobalt phosphide as a highly active non-precious metal cocatalyst for photocatalytic hydrogen production under visible light irradiation. J. Mater. Chem. A 3:6096-6101. doi: 10.1039/C4TA07149B

Cao, S., Chen, Y., Wang, C. J., He, P., and Fu, W. F. (2014). Highly efficient photocatalytic hydrogen evolution by nickel phosphide nanoparticles from aqueous solution. Chem. Commun. 50, 10427-10429. doi: $10.1039 / \mathrm{C} 4 \mathrm{CC} 05026 \mathrm{~F}$

Chai, Z., Zeng, T. T., Li, Q., Lu, L. Q., Xiao, W. J., and Xu, D. (2016). Efficient visible light-driven splitting of alcohols into hydrogen and corresponding carbonyl compounds over a Ni-modified CdS photocatalyst. J. Am. Chem. Soc. 138, 10128-10131. doi: 10.1021/jacs.6b06860

Chen, Y., Zhao, S., Wang, X., Peng, Q., Lin, R., Wang, Y., et al. (2016). Synergetic integration of $\mathrm{Cu}_{1.94} \mathrm{~S}-\mathrm{Zn}_{\mathrm{x}} \mathrm{Cd}_{1-\mathrm{X}} \mathrm{S}$ heteronanorods for enhanced visible-lightdriven photocatalytic hydrogen production. J. Am. Chem. Soc. 138, 4286-4289. doi: 10.1021 /jacs.5b12666

Cheng, H., Lv, X. J., Cao, S., Zhao, Z. Y., Chen, Y., and Fu, W. F. (2016). Robustly photogenerating $\mathrm{H}_{2}$ in water using $\mathrm{FeP} / \mathrm{CdS}$ catalyst under solar irradiation. Sci. Rep. 6:19846. doi: 10.1038/srep19846

Concepcion, J. J., Jurss, J. W., Brennaman, M. K., Hoertz, P. G., Patrocinio, A. O., Iha, N. Y. M., et al. (2009). Making oxygen with ruthenium complexes. Acc. Chem. Res. 42, 1954-1965. doi: 10.1021/ar9001526

Costi, R., Saunders, A. E., Elmalem, E., Salant, A., and Banin, U. (2008). Visible light-induced charge retention and photocatalysis with hybrid CdSe-Au nanodumbbells. Nano Lett. 8, 637-641. doi: 10.1021/nl0730514

Ding, T. X., Olshansky, J. H., Leone, S. R., and Alivisatos, A. P. (2015). Efficiency of hole transfer from photoexcited quantum dots to covalently linked molecular species. J. Am. Chem. Soc. 137, 2021-2029. doi: 10.1021/ja512278a

Fuhr, A. S., Yun, H. J., Makarov, N. S., Li, H., McDaniel, H., and Klimov, V. I. (2017). Light emission mechanisms in $\mathrm{CuInS}_{2}$ quantum dots evaluated by spectral electrochemistry. ACS Photonics 4, 2425-2435. doi: 10.1021/acsphotonics.7b00560

Garakyaraghi, S., and Castellano, F. N. (2018). Nanocrystals for triplet sensitization: molecular behavior from quantum-confined materials. Inorg. Chem. 57, 2351-2359. doi: 10.1021/acs.inorgchem.7b03219

Grenland, J. J., Maddux, C. J. A., Kelley, D. F., and Kelley, A. M. (2017). Charge trapping versus exciton delocalization in CdSe quantum dots. J. Phys. Chem. Lett. 8, 5113-5118. doi: 10.1021/acs.jpclett.7b02242

Han, Z., Qiu, F., Eisenberg, R., Holland, P. L., and Krauss, T. D. (2012). Robust photogeneration of $\mathrm{H}_{2}$ in water using semiconductor nanocrystals and a nickel catalyst. Science 338, 1321-1324. doi: 10.1126/science.1227775

Hara, M., Waraksa, C. C., Lean, J. T., Lewis, B. A., and Mallouk, T. E. (2000). Photocatalytic water oxidation in a buffered tris(2,2'-bipyridyl)ruthenium complex-colloidal $\mathrm{IrO}_{2}$ system. J. Phys. Chem. A 104, 5275-5280. doi: $10.1021 /$ jp000321x

Hewa-Kasakarage, N. N., Kirsanova, M., Nemchinov, A., Schmall, N., El-Khoury, P. Z., Tarnovsky, A. N., et al. (2009). Radiative recombination of spatially extended excitons in (ZnSe/CdS)/CdS heterostructured nanorods. J. Am. Chem. Soc. 131, 1328-1334. doi: 10.1021/ja8082895

Hines, D. A., and Kamat, P. V. (2014). Recent advances in quantum dot surface chemistry. ACS Appl. Mater. Interfaces 6, 3041-3057. doi: 10.1021/am405196u

Hofbeck, T., and Yersin, H. (2010). The triplet state of fac-Ir(ppy) 3. Inorg. Chem. 49, 9290-9299. doi: $10.1021 /$ ic $100872 \mathrm{w}$

Jensen, S. C., Bettis Homan, S., and Weiss, E. A. (2016). Photocatalytic conversion of nitrobenzene to aniline through sequential proton-coupled one-electron transfers from a cadmium sulfide quantum dot. J. Am. Chem. Soc. 138, 1591-1600. doi: 10.1021/jacs.5b11353

Kalisman, P., Kauffmann, Y., and Amirav, L. (2015). Photochemical oxidation on nanorod photocatalysts. J. Mater. Chem. A 7, 3261-3265. doi: 10.1039/C4TA06164K
Kalisman, P., Nakibli, Y., and Amirav, L. (2016). Perfect photonto- hydrogen conversion efficiency. Nano Lett. 16, 1776-1781. doi: $10.1021 /$ acs.nanolett.5b04813

Kamat, P. V., Christians, J. A., and Radich, J. G. (2014). Quantum dot solar cells: hole transfer as a limiting factor in boosting the photoconversion efficiency. Langmuir 30, 5716-5725. doi: 10.1021/la500555w

Kholmicheva, N., Moroz, P., Bastola, E., Razgoniaeva, N., Bocanegra, J., Shaughnessy, M., et al. (2015). Mapping the exciton diffusion in semiconductor nanocrystal solids. ACS Nano 9, 2926-2937. doi: 10.1021/nn507322y

Kholmicheva, N., Razgoniaeva, N., Yadav, P., Lahey, A., Erickson, C., Moroz, P., et al. (2017). Enhanced emission of nanocrystal solids featuring slowly diffusive excitons. J. Phys. Chem. C 121, 1477-1487. doi: 10.1021/acs.jpcc.6b10994

Khon, E., Lambright, K., Khnayzer, R. S., Moroz, P., Perera, D. N., Butaeva, E., et al. (2013). Improving the catalytic activity of semiconductor nanocrystals through selective domain etching. Nano Lett. 13, 2016-2023. doi: 10.1021/nl400715n

Khon, E., Mereshchenko, A., Tarnovsky, A. N., Acharya, K., Klinkova, A., Hewa-Kasakarage, N. N., et al. (2011). Suppression of the plasmon resonance in $\mathrm{Au} / \mathrm{CdS}$ colloidal nanocomposites. Nano Lett. 11, 1792-1799. doi: $10.1021 / \mathrm{nl} 200409 \mathrm{x}$

Knowles, K. E., Nelson, H. D., Kilburn, T. B., and Gamelin, D. R. (2015). Singlet-triplet splittings in the luminescent excited states of colloidal $\mathrm{Cu}^{+}: \mathrm{CdSe}, \mathrm{Cu}^{+}: \mathrm{InP}$, and $\mathrm{CuInS}_{2}$ nanocrystals: charge transfer configurations and self-trapped excitons. J. Am. Chem. Soc. 137, 13138-13147. doi: $10.1021 /$ jacs.5b08547

Leach, A. D. P., and Macdonald, J. E. (2016). Optoelectronic properties of $\mathrm{CuInS}_{2}$ nanocrystals and their origin. J. Phys. Chem. Lett. 7, 572-583. doi: $10.1021 /$ acs.jpclett.5b02211

Lian, S., Weinberg, D. J., Harris, R. D., Mohamad, S., Kodaimati, M. S., and Weiss, E. A. (2016). Subpicosecond photoinduced hole transfer from a CdS quantum dot to a molecular acceptor bound through an exciton-delocalizing ligand. ACS Nano 10, 6372-6382. doi: 10.1021/acsnano.6b02814

Liu, C., Colon, B. C., Ziesack, M., Silver, P. A., and Nocera, D. G. (2016). Water splitting-biosynthetic system with $\mathrm{CO}_{2}$ reduction efficiencies exceeding photosynthesis. Science 352, 1210-1213. doi: 10.1126/science.aaf5039

McCusker, C. E., and Castellano, F. N. (2016). Materials integrating photochemical upconversion. Top. Curr. Chem. 374, 1-25. doi: 10.1007/s41061-016-0021-7

Mongin, C., Garakyaraghi, S., Razgoniaeva, N., Zamkov, M., and Castellano, F. N. (2016). Direct observation of triplet energy transfer from semiconductor nanocrystals. Science 351, 369-372. doi: 10.1126/science.aad6378

Najafpour, M. M., Heidari, S., Balaghi, S. E., Hołynska, M., Sadr, M. H., Soltani, B., et al. (2017). Proposed mechanisms for water oxidation by Photosystem II and nanosized manganese oxides. Biochim. Biophys. Acta Bioenerg. 1858, 156-174. doi: 10.1016/j.bbabio.2016.11.007

Nakibli, Y., Kalisman, P., and Amirav, L. (2015). Less is more: the case of metal cocatalysts. J. Phys. Chem. Lett. 6, 2265-2268. doi: $10.1021 /$ acs.jpclett.5b00872

Nelson, H. D., Hinterding, S. O. M., Fainblat, R., Creutz, S. E., Li, X., and Gamelin, D. R. (2017). Mid-gap states and normal vs inverted bonding in luminescent $\mathrm{Cu}^{+}$- and $\mathrm{Ag}^{+}$-doped CdSe nanocrystals. J. Am. Chem. Soc. 139, 6411-6421. doi: $10.1021 /$ jacs.7b01924

O'Connor, T., Panov, M. S., Mereshchenko, A., Tarnovsky, A. N., Lorek, R., Perera, D., et al. (2012). The effect of the charge-separating interface on exciton dynamics in photocatalytic colloidal heteronanocrystals. ACS Nano 6 , 8156-8165. doi: $10.1021 / \mathrm{nn} 302810 \mathrm{y}$

Olshansky, J. H., Ding, T. X., Lee, Y. V., Leone, S. R., and Alivisatos, A. P. (2015). Hole transfer from photoexcited quantum dots: the relationship between driving force and rate. J. Am. Chem. Soc. 137, 15567-15575. doi: 10.1021/jacs.5b10856

Perera, D., Lorek, R., Khnayzer, R. S., Moroz, P., O'Connor, T., Khon, D., et al. (2012). Photocatalytic activity of core/shell semiconductor nanocrystals featuring spatial separation of charges. J. Phys. Chem. C 116, 22786-22793. doi: $10.1021 /$ jp308921s

Pu, C., Ma, J., Qin, H., Yan, M., Fu, T., Niu, Y., et al. (2016). Doped semiconductornanocrystal emitters with optimal photoluminescence decay dynamics in microsecond to millisecond range: synthesis and applications. ACS Cent. Sci. 2, 32-39. doi: 10.1021/acscentsci.5b00327

Raza, F., Yim, D., Park, J. H., Kim, H. I., Jeon, S. J., and Kim, J. H. (2017). Structuring Pd nanoparticles on $2 \mathrm{H}$-WS2 nanosheets induces excellent 
photocatalytic activity for cross-coupling reactions under visible light. J. Am. Chem. Soc. 139, 14767-14774. doi: 10.1021/jacs.7b08619

Schultz, D. M., and Yoon, T. P. (2014). Solar synthesis: prospects in visible light photocatalysis. Science 343:1239176. doi: 10.1126/science.1239176

Simon, T., Bouchonville, N., Berr, M. J., Vaneski, A., Adrović, A., and Volbers, D., et al. (2014). Redox shuttle mechanism enhances photocatalytic H2 generation on Ni-decorated CdS nanorods. Nat. Mater 13, 1013-1018. doi: 10.1038/nmat4049

Twilton, J., Le, C., Zhang, P., Shaw, M. H., Evans, R. W., and MacMillan, D. W. C. (2017). The merger of transition metal and photocatalysis. Nat. Rev. Chem. 1:0052. doi: 10.1038/s41570-017-0052

Utterback, J. K., Grennell, A. N., Wilker, M. B., Pearce, O., Eaves, J. D., and Dukovic, G. (2016). Observation of trapped-hole diffusion on the surfaces of CdS nanorods. Nat. Chem. 8, 1061-1066. doi: 10.1038/nchem.2566

Warren, S. C., Voïtchovsky, K., Dotan, H., Leroy, C. M., Cornuz, M., Stellacci, F., et al. (2013). Identifying champion nanostructures for solar water-splitting. Nat. Mater. 12, 842-849. doi: 10.1038/nmat3684

Wu, K., Du, Y., Tang, H., Chen, Z., and Lian, T. (2015). Efficient extraction of trapped holes from colloidal CdS nanorods. J. Am. Chem. Soc. 137, 10224-10230. doi: 10.1021/jacs.5b04564

Wu, M., Congreve, D. N., Wilson, M. W. B., Jean, J., Geva, N., Welborn, M., et al. (2016). Solid-state infrared-to-visible upconversion sensitized by colloidal nanocrystals. Nat. Photon. 10, 31-34. doi: 10.1038/nphoton.2015.226

Yan, Y., Crisp, R., Gu, J., Chernomordik, B., Pach, G., Marshall, A., et al. (2017). Multiple exciton generation for photoelectrochemical hydrogen evolution reactions with quantum yields exceeding 100\%. Nat. Energy 2:17052. doi: 10.1038/nenergy.2017.52

Yang, H. B., Miao, J., Hung, S. F., Huo, F., Chen, H. M., and Liu, B. (2014). Stable quantum dot photoelectrolysis cell for unassisted visible light solar water splitting. ACS Nano 8, 10403-10413. doi: 10.1021/nn503751s

Yu, W. W., Qu, L., Guo, W., and Peng, X. (2003). Experimental determination of the extinction coefficient of CdTe, CdSe, and CdS nanocrystals. Chem. Mater. 15, 854-2860. doi: $10.1021 / \mathrm{cm} 034081 \mathrm{k}$
Yu, X., An, X., Shavel, A., Ibáñez, M., and Cabot, A. (2014b). The effect of the Ga content on the photocatalytic hydrogen evolution of $\mathrm{CuIn}_{1}$ ${ }_{\mathrm{x}} \mathrm{Ga}_{\mathrm{x}} \mathrm{S}_{2}$ nanocrystals. J. Mater. Chem. A 2, 12317-12322. doi: 10.1039/C4TA0 $1315 \mathrm{H}$

Yu, X., Shavel, A., An, X., Luo, Z., Ibáñez, M., and Cabot, A. (2014a). $\mathrm{Cu}_{2} \mathrm{ZnSnS}_{4}$ $\mathrm{Pt}$ and $\mathrm{Cu}_{2} \mathrm{ZnSnS}_{4}-\mathrm{Au}$ heterostructured nanoparticles for photocatalytic water splitting and pollutant degradation. J. Am. Chem. Soc. 136, 9236-9239. doi: $10.1021 / j a 502076 \mathrm{~b}$

Zamkov, M. (2017). Solar hydrogen generation: exceeding 100\% efficiency. Nat. Energy 2:17072. doi: 10.1038/nenergy.2017.72

Zhou, Y., Hu, W., Ludwig, J., and Huang, J. (2017). Exceptionally robust $\mathrm{CuInS}_{2} / \mathrm{ZnS}$ nanoparticles as single component photocatalysts for $\mathrm{H} 2$ evolution. J. Phys. Chem. C 121, 19031-19035. doi: 10.1021/acs.jpcc.7b 05241

Zhu, H., Song, N., Lv, H., Hill, C. L., and Lian, T. (2012). Near unity quantum yield of light-driven redox mediator reduction and efficient $\mathrm{H}_{2}$ generation using colloidal nanorod heterostructures. J. Am. Chem. Soc. 134, 11701-11708. doi: $10.1021 / \mathrm{ja} 303698 \mathrm{e}$

Zhukovskyi, M., Tongying, P., Yashan, H., Wang, Y., and Kuno, M. (2015). Efficient photocatalytic hydrogen generation from $\mathrm{Ni}$ nanoparticle decorated CdS nanosheets. ACS Catal. 5, 6615-6623. doi: 10.1021/acscatal.5b01812

Conflict of Interest Statement: The authors declare that the research was conducted in the absence of any commercial or financial relationships that could be construed as a potential conflict of interest.

Copyright (C) 2018 Moroz, Boddy and Zamkov. This is an open-access article distributed under the terms of the Creative Commons Attribution License (CC BY). The use, distribution or reproduction in other forums is permitted, provided the original author(s) and the copyright owner(s) are credited and that the original publication in this journal is cited, in accordance with accepted academic practice. No use, distribution or reproduction is permitted which does not comply with these terms. 